\title{
VALOR DE MERCADO DE PLANTACIONES DE TECA (Tectona grandis Linn.) EN EL DEPARTAMENTO DE CÓRDOBA
}

\section{MARKET VALUE OF TEAK PLANTATIONS (Tectona grandis Linn.) IN THE DEPARTMENT OF CORDOBA}

\author{
Cesar A. Vergara Córdoba ${ }^{*}$, Carlos E. Cardona Ayala, Olman Murillo Gamboa², Alfredo \\ D. Jarma Orozco ${ }^{1}$, Hermes Araméndiz Tatis ${ }^{1}$
}

Recibido para publicación: Julio 16 de 2012 - Aceptado para publicación: Abril 18 de 2013

\begin{abstract}
RESUMEN
La teca (Tectona grandis L.) presenta gran estabilidad en ambientes cambiantes y es resistente a la degradación de agentes bióticos y abióticos. Por ello, su madera es considerada como una de las más valiosas del mundo. El objetivo de este estudio fue estimar el valor de madera en pie de teca en Córdoba, Colombia. Se utilizaron parcelas circulares permanentes de $500 \mathrm{~m} 2$ con una intensidad de muestreo de $2 \%$, en cinco rodales comerciales en los municipios de San Antero y Canalete, Córdoba. Se estimó el valor real en pie en función de la edad, calidad y dimensión de trozas, volumen (por clase diamétrica), potencial de aprovechamiento y transporte. Los resultados mostraron que la plantación El Limón (San Antero), con el más alto volumen de madera en pie, fue la de que más disminuyó su valoración $(67,5 \%)$. La plantación mejor valorada fue Guazimal (Canalete), con una disminución de su valor real de 56,7\%. El grosor de los árboles fue el factor que mayor influenció en la disminución del valor, lo que amerita una mejora en la selección del material de siembra y en el manejo silvícola. Este trabajo muestra el valor actual de las plantaciones de teca en el departamento de Córdoba y servirá de punto de referencia para futuras investigaciones.
\end{abstract}

Palabras clave: plantaciones forestales, reforestación, silvicultura, teca.

\begin{abstract}
Teak (Tectona grandis L.) has great stability in changing environments and is resistant to degradation by biotic and abiotic agents. For these reasons, its wood is considered one of the most valuable in the world. The objective of this study was to estimate the value of teak timber in Cordoba, Colombia. Permanent circular plots of $500 \mathrm{~m} 2$ with $2 \%$, of sampling intensity in five commercial stands in San Antero and Canalete, Córdoba, were used. The commercial value of the potential timber production in each plot were estimated based on
\end{abstract}

\footnotetext{
'Universidad de Córdoba, Departamento de Ingeniería Agronómica y Desarrollo Rural, Carrera 6 No 76-103, Montería-Córdoba. Telefax (4) 786 0255. E-mail:ceveco@fca.edu.co, cardona@fca.edu.co, jarma@fca.edu.co, aramendiz@fca.edu.co

2Instituto Tecnológico de Costa Rica. olmuga@yahoo.es
} 
the age, quality and size of logs, volume (by diameter class), potential use and transport. The results showed that trees at the field El Limon (San Antero), with the highest volume of timber production, had the lowest commercial value (67.5\%). The highest commercial value was found in Guazimal (Canalete), with a decrease in the real value of $56.7 \%$. The diameter of the trees was the most important factor on the commercial value of the trees, this suggest that it is very important to improve the selection of the planting material and the management of the crop. This paper shows the current value of teak plantations in the department of Córdoba and it will be as a reference point for future research in this area.

Key words: forest plantations, reforestation, silviculture, teak.

\section{INTRODUCCIÓN}

En Colombia,a lo largo de 16 años, se han establecido 173.950 hectáreas de bosque, gracias a la incentivación económica que genera el Certificado de Incentivo Forestal (CIF), como política de participación ciudadanaa nivel nacional, incrementando los beneficios ambientales y sociales. El Gobierno Nacional ha invertido \$251.758 millones en el CIF, entre 1995 y 2011 (Ordóñez 2012).

En el departamento de Córdoba el área de aptitud forestal corresponde al 40\% (897.086 ha) de su superficie, las principales especies plantadas con su porcentaje en relación al área dedicada a plantaciones forestales (28.500 ha) son: Tabebuia rosea (34,83\%), Tectona grandis (25,78\%), Acacia mangium (24,9\%) y Bombacopsis quinata $(7,78 \%)$, lo que indica, que la especie objeto de estudio de este trabajo abarcan una superficie de 7.347 ha en el departamento de Córdoba (Rincón 2009).

La comercialización de la madera es un tema poco tratado en Colombia y los productores desconocen como estimar el valor de su plantación en pie, de tal forma que les permita comercializar su madera de la mejor manera para obtener mayor beneficio económico, por otro lado los comerciantes no tienen unificados los criterio a la hora de comprar una plantación esto se evidencia en estudios realizados en Colombia por la Pontificia Universidad Javeriana (Devia 2000).

Es necesario para la cadena forestal buscar alternativas viables para incorporar el valor de la madera, esta valoración permitiría contar con proyecciones administrativas y manejo de las áreas boscosas (naturales o plantadas), así mismo plantearía un reto a la necesidad de optimizar el uso del recurso forestal, mejorar tecnología, ajustar procesos productivos y de comercialización. Se debe avanzar sobre inventarios forestales detallados y ajuste a la valoración propuesta para la madera, que permitan definir datos más confiables y valorar realmente los bosques, además de facilitar la formulación de los Planes de Manejo Forestal (Devia 2003); teniendo en cuenta que los cálculos proyectados de la demanda mundial de madera para el año 2040 indican que podría llegar hasta los 20 millones de metros cúbicos anuales (CONFEMADERAS 2011).

La teca (Tectona grandis L.), también conocida como teak (India, Tailandia, Birmania e Indonesia), teck (Francia), ojati (Java), maysak (Laos) e tiek (Alemania) es una especie arbórea de la familia Verbenaceae que tiene un alto valor comercial. El producto principal de 
esta especie es la madera, muy utilizada en carpintería, producción de muebles finos, y especialmente en el sector de la construcción naval, donde es prácticamente insustituible, porque resiste el sol, el calor, el frío y la lluvia y el agua de mar. La combinación de belleza, resistencia y durabilidad de la madera de esta especie es lo que la ha convertido en una de las más valiosas del mundo (Matricardi 1989; Lamprecht 1990; Rondón et al. 1998; Oliveira 2003; Arruda et al. 2003).

Teóricamente la valuación de objetos o bienes se ha venido realizando desde tiempo inmemorial como una necesidad de la humanidad surgida de manera espontánea para permitir el intercambio. Desde el establecimiento de los primeros asentamientos urbanos, que se remontan a la antigua Babilonia, y posteriormente, al introducirse el derecho absoluto de propiedad en el imperio romano (460 A.C.), se da por primera vez la práctica común de establecer previamente valores sobre objetos, bienes raíces y derechos sobre la figura jurídica de la propiedad privada (Arechederra 2010).

La valuación es una actividad indispensable que sirve a particulares, instituciones de crédito y empresas en general, para facilitar la toma de decisiones en sus proyectos financieros, en cuanto a lograr una mejor aplicación de sus recursos disponibles en operaciones relacionadas con el bien, ya sea de compra o venta, de créditos, de efectos fiscales o contables, de seguro, entre otros (Silva et al. 2010).

Valuar un bien económico, como en el caso de un predio agrícola, ganadero, o forestal es atribuirle un valor a partir de los fines para los cuales se realiza el avalúo, y de las características físicas, económicas y de producción (Berlanga et al. 2004). Según la definición de Caballer (1999): "La valoración es aquella parte de la economía cuyo objeto es la estimación de un determinado valor o varios valores con arreglo a unas determinadas hipótesis, con vistas a unos fines determinados y mediante procesos de cálculo basados en informaciones de carácter técnico".

El cálculo de la oferta de madera de la base forestal actual para abastecimiento futuro es una de las primeras acciones en la implementación de un plan de contingencia ante una posible situación de desabastecimiento de madera (Corella 2009). La variación de las propiedades de la madera dentro del árbol a lo largo del radio y desde la base al ápice está asociada a la formación de madera juvenil, que ha sido objeto de estudio mayor en coníferas que en latifoliadas; teniendo en cuenta que la madera juvenil posee menores propiedades de importancia económica, como la densidad y longitud de fibras, que produce pulpas de inferior calidad y rendimiento (Barnett $y$ Jeronimidis 2003).

Según Murillo y Badilla (2004) el Colegio de Ingenieros Agrónomos de Costa Rica, ha establecido un programa de formación y capacitación de peritos en este campo, para poder atender la demanda de servicios profesionales de este tipo en el sector agropecuario. Es así como los autores desarrollaron una metodología que permite estimar el valor real de las plantaciones forestales, basado no solo en el volumen y dimensiones de los árboles, sino también en su calidad en relación con su potencial de 
producción de madera comercial.

Las dimensiones de la troza, en un árbol, al igual que características como altura son altamente heredables, según lo reportado por Espitia et al. (2010), quienes, en Acacia mangium, registraron ganancias genéticas esperadas en promedio de árboles plus A del 22,24\% y el $48,57 \%$ en altura y volumen comercial, respectivamente; Vallejos et al. (2010), a su vez, relacionan ganancias genéticas esperadas, en general, entre 20-25\% en volumen; Mesén (2001), quien estimó ganancias genéticas en melina del $17 \%$ en altura y el $43 \%$ en DAP; Cornelius \& Hernández (1994), en la misma especie, reportaron ganancias genéticas de hasta un $12 \%$ en rectitud del fuste; Kumar et al. (2006), en melina en el ámbito clonal, encontraron para altura, diámetro basal y diámetro a la altura del pecho, ganancias de un $18 \%$, $25 \%$ y $30 \%$; Rojas y Arias (2004).

Según Murillo y Badilla (2004) en un futuro, el diámetro de referencia del precio de la madera en pie se irá aproximando a los diámetros de $40 \mathrm{~cm}$ a medida que el mejoramiento genético consiga obtener individuos de mayor crecimiento y rendimiento en plantación.

En Córdoba no se registran antecedentes relacionados con la valoración de plantaciones forestales. El objetivo de este trabajo fue estimar el valor en pie de teca en el departamento de Córdoba - Colombia, con miras a implementar esta metodología en producción forestal del departamento.

\section{MATERIALES Y MÉTODOS}

La presente investigación se desarrolló en el año 2011, utilizando los datos de las parcelas permanentes de cinco rodales comerciales de teca (Tectona grandis Linn) instaladas por la Universidad deCórdoba en dos núcleosforestales (San Antero y Canalete) en el departamento de Córdoba (Colombia) (tabla 1). El trabajo fue realizado con financiación del Ministerio de Agricultura y Desarrollo Rural de Colombia, como parte de los programas y proyectos de investigación, desarrollo tecnológico e innovación para el sector agropecuario por cadenas productivas, del año 2007.

Tabla 1. Condiciones agroecológicas de los núcleos forestales de la investigación.

\begin{tabular}{lll}
\hline Condiciones agroecológicas & \multicolumn{1}{c}{ Canalete } & \multicolumn{1}{c}{ San Antero } \\
\hline Precipitación media anual & $1.000-1.500 \mathrm{~mm}$ & $1.320 \mathrm{~mm}$ \\
Períodos secos & Diciembre-marzo & Noviembre - marzo \\
Períodos húmedos & Marzo - octubre & Marzo-octubre \\
Meses más lluviosos & Julio y octubre & Julio y octubre \\
Temperatura promedio & $27,0^{\circ} \mathrm{C}$ & $27,4^{\circ} \mathrm{C}$ \\
Temperatura máxima & $28,4^{\circ} \mathrm{C}$ & $28,4^{\circ} \mathrm{C}$ \\
Temperatura mínima & $25,6^{\circ} \mathrm{C}$ & $26,6^{\circ} \mathrm{C}$ \\
Rango altitudinal & 67 m.s.n.m. & 25 m.s.n.m \\
Formación Ecológica & Bs-T & Bs-T \\
\hline
\end{tabular}

Fuente: Plan de Ordenamiento territorial de cada municipio. Bs-T = Bosque seco Tropical 
La metodología a seguir para la recolección de información es la publicada por Murillo y Badilla (2004) y se explica a continuación.

Descripción de las parcelas permanentes: Las parcelas permanentes circulares de $500 \mathrm{~m}^{2}$ se establecieron con una intensidad de muestreo del $2 \%$, ubicadas en forma sistemática en fajas cada 100 metros, según el procedimiento estándar propuesto por Murillo y Camacho (1997) y Murillo y Badilla (2004). Dentro de la parcela se midió en cada árbol las siguientes variables: altura total $(\mathrm{m})$, diámetro a la altura de pecho DAP $(\mathrm{cm})$, rectitud del fuste, ángulo de las ramas, presencia de bifurcaciones, de reiteraciones, de grano en espiral, de gambas, inclinación del fuste y estado fitosanitario. Con base en todos estos criterios, se calificó la calidad general de cada una de las primeras 4 trozas (de 2,5 m de largo).

La calidad del árbol se obtuvo con base en la calidad de sus primeras cuatro trozas, ajustadas por un peso económico de 40\%,30\%, 20\% y $10 \%$ según su posición dentro del árbol, de abajo hacia arriba respectivamente. El principio se basa en poder determinar en pie, el potencial para producir madera de aserrío de cada individuo o de sus trozas, en una escala de calificación de 1 a 4, donde 1 es el mayor valor (Murillo y Camacho 1998; Murillo 1999).

El volumen de cada una de las trozas, se obtuvo con la siguiente ecuación:

$$
V=\left(\frac{D+d}{2}\right)^{2} * \Pi / 4 * L
$$

Dónde: $\mathrm{D}=$ Diámetro mayor $(\mathrm{cm}) ; \mathrm{d}=$ Diámetro menor $(\mathrm{cm})$ y $\mathrm{L}=$ Longitud $(2,5 \mathrm{~m}$ para cada troza).
La estimación del valor real en pie de cada plantación forestal, se determinó la distribución del volumen según sus dimensiones (clases diamétricaen la cara menor), calidad, edad y la especie.

Valor Real en pie $=$ Especie + Edad + Calidad + Volumen (clase diamétrica)

\section{Precio del $\mathbf{m}^{3}$ de madera en pie según la especie:} El precio de la madera en Colombia no está regulado por ninguna entidad, de tal manera que en este caso, para estimar el valor de las plantaciones en pie, se realizaron consultas a comerciantes de madera y se calculó un valor para ser utilizado en la valoración de las plantaciones en pie.

\section{Ajuste del valor en pie por la edad del árbol: $\mathrm{El}$} mercado de la madera de teca tiende a pagar un mejor precio por la madera adulta (mayor peso específico y mejores propiedades) y con duramen, que por la madera juvenil y con albura. Se ajustó entonces el valor real en pie con base en la edad como sigue:

Valor real inicial * 1 (si es mayor a 10 años de edad) y Valor real inicial * 0,8 (si la plantación es menor a 10 años de edad). Así, se obtuvo un nuevo estimado de valor real de la plantación en pie, con base en la sumatoria de todos los nuevos valores ajustados por la edad.

Ajuste del valor en pie por la calidad de la troza: La estimación del valor real inicial se ajustó según la calidad de las trozas en pie:

Valor real inicial * 1,0 (trozas de calidad 1); Valor real inicial * 0,9 (trozas de calidad 2); Valor real inicial * 0,8 (trozas de calidad 3) y Valor real inicial $* 0$ (trozas de calidad 4$)$. Se 
consideran que no tienen valor comercial para aserrío. Se obtuvo entonces un nuevo estimado de valor real de la plantación en pie, con base en la sumatoria de todos los nuevos valores ajustados por su calidad.

\section{Ajuste del valor en pie por las dimensiones} de la troza: El porcentaje de rendimiento en aserrío que se obtiene de una troza de madera, está principalmente, en función de su calidad y dimensiones. Por lo tanto, una plantación con $100 \mathrm{~m}^{3}$ en pie, cuyos diámetros oscilan de 10 a $20 \mathrm{~cm}$, no podrá tener el mismo valor que una plantación con el mismo volumen, pero con árboles de 30 a $40 \mathrm{~cm}$ de diámetro. Con base en un estimado general del porcentaje de rendimiento en aserrío vs dimensiones de la troza, desarrollado por el proyecto COSEFORMA en la zona norte de Costa Rica (Corrales 1996), se realizó un ajuste al modelo, donde se estima que los precios actuales del mercado de la madera en pie de plantaciones, se basan en trozas de poco más de 10 pulgadas madereras o unos $25 \mathrm{~cm}$ de DAP. Por encima de estos $25 \mathrm{~cm}$ en la cara menor, por lo general es considerado como madera gruesa por el mercado.

El ajuste por clase diamétrica se realizó como sigue (Murillo y Badilla 2004):

Valor inicial $* 1,0$ (para todos los individuostrozas diámetro $\geq 22,5$ y $<27,5 \mathrm{~cm}$ )

Valor inicial $* 0,90$ (para todos los individuostrozas diámetro $<22,5$ y $\geq 17,5 \mathrm{~cm}$ )

Valor inicial * 0,73 (para todos los individuostrozas diámetro $<17,5 \mathrm{y} \geq 12,5 \mathrm{~cm})$

Valor inicial * 0,48 (para todos los individuostrozas diámetro $<12,5 \mathrm{~cm})$

Valor inicial * 1,04 (para todos los individuostrozas diámetro $\geq 27,5$ y $<32,5 \mathrm{~cm}$ )
Valor inicial $* 1,06$ (para todos los individuostrozas diámetro $\geq 32,5$ y $<37,5 \mathrm{~cm}$ )

Valor inicial $* 1,08$ (para todos los individuostrozas diámetro $\geq 37,5$ y $<42,5 \mathrm{~cm}$ )

Valor inicial $* 1,09$ (para todos los individuostrozas diámetro $\geq 42,5$ y $<47,5 \mathrm{~cm}$ )

Valor inicial $* 1,10$ (para todos los individuostrozas diámetro $\geq 47,5 \mathrm{~cm}$ )

Se obtuvo entonces el valor real en pie de la plantación, con base en la sumatoria de todos los valores generados.

\section{Ajuste del valor real en pie por el potencial de aprovechamiento y transporte: El valor de mercado en pie se estimó de acuerdo a los siguientes criterios (Murillo et al. 2004).}

\section{Criterios de mercado que pueden afectar al valor real de la plantación forestal: Potencial de aprovechamiento $\mathrm{y}$ transporte $=$ escala de operaciones + acceso dentro de finca + distancia a industria + topografía y pendiente del terreno + densidad de plantación + presencia de malezas + presencia de pedregosidad.}

\section{Escala de operaciones o tamaño de la plantación:}

El tamaño de la plantación influirá decididamente en su valor por efecto en los costos de manejo y aprovechamiento. A mayor escala de producción menores costos de aprovechamiento.

Se usó entonces la siguiente escala de valoración: Escala 1, plantaciones < a 5 ha (60\% del valor real): Escala 2, plantaciones de $5-20$ ha $(80 \%$ del valor real) y Escala 3, plantaciones > a 20 ha (100\% del valor real).

Acceso a la plantación: La valoración se realizó con la siguiente escala: 
Acceso: 1) Aquellas plantaciones con acceso todo el año con vehículos livianos hasta el borde mismo. Los camiones pueden Ilegar hasta el borde mismo de la plantación para efectuar la carga de la madera. Se le otorga un $100 \%$ del valor real. 2) aquellas plantaciones que requieren labores leves de acondicionamiento de caminos en una distancia menor a un $1 \mathrm{~km}$ desde la entrada de la finca. Se le asignó un $65 \%$ del valor real. 3) plantaciones que requieren de movimientos grandes de tierra y apertura de caminos en una distancia mayor a $1 \mathrm{~km}$. Se le asignó un 30\% del valor real de la plantación.

Distancia al sitio de transformación: La distancia hasta la industria de transformación de la madera afecta sensiblemente los costos y estrategia de transporte requerido. Este criterio se analiza e interpreta como sigue: Distancia 1: de 0-25 km. Se le asignó un 100\% del valor real; Distancia 2: de 25-100 km. Se le asignó un $80 \%$ del valor real y Distancia 3: $>100 \mathrm{~km}$. Se le asignó un $60 \%$ del valor real.

Pedregosidad: La pedregosidad excesiva en una plantación provoca la partidura de muchos árboles durante su aprovechamiento. Estos individuos pierden entonces su valor económico. La valoración se realizó con la siguiente escala:

1: con ausencia de pedregosidad que afecte el aprovechamiento y cause daños mecánicos a los árboles. Se le asignó el 100\% del valor real y 2: con afloramientos rocosas grandes que puedan causar daños mecánicos o partiduras a los árboles cortados. Se le asignó el 85\% del valor real.

Densidad de la plantación: Las plantaciones con una alta densidad de individuos por hectárea aumentan considerablemente los costos, rendimientos y riesgos de aprovechamiento. El aumento en la frecuencia de árboles que no caen fácilmente y se apoyan en otros, los que se sostienen con lianas, los que se acumulan encima de otros, es el resultado inmediato. Este criterio se calificó como sigue: 1: aquellas plantaciones con menos de 400 a 500 árboles ha $^{-1}$. Se le asignó un $100 \%$ de su valor real y 2 : aquellas plantaciones con más de 500 árboles $\mathrm{ha}^{-1}$, se les penalizó con un $85 \%$ del valor real.

Presencia de malezas: Las plantaciones con excesiva maleza no permiten un aprovechamiento rápido y eficiente. Los obreros tienen que invertir tiempo en la limpieza de cada uno de los árboles a tumbar. Por tanto, este criterio se calificó como sigue: 1: cuando las malezas del terreno no impiden el aprovechamiento de manera significativa y se puede transitar libremente por la plantación sin necesidad de abrirse paso. Se le asignó un $100 \%$ del valor real y 2 : cuando es necesario hacer labores de limpieza para poder hacer el aprovechamiento. Se le asignó un 85\% del valor real de la plantación.

Topografía y pendiente: Este es quizá uno de los criterios que mayor influencia tienen en los costos y estrategia de aprovechamiento de plantaciones forestales. El criterio se evaluó como se describe a continuación:

1) En los sitios donde la pendiente es ligera de $0-15 \%$, o que presentaba lomas largas con ausencia de muchos obstáculos (zonas fangosas o cañadas muy pronunciadas). Se le asignó un 100\% del valor real. 2) plantaciones que presentaron pendientes del 15-30\% o 
lomas cortas y pronunciadas. Se le asignó un $65 \%$ del valor real. 3) plantaciones con pendientes mayores al 30\%, y presencia de zonas fangosas o riachuelos entre el acceso más cercano para su extracción o lomas cortas y abruptas. Se le asignó un 30\% del valor real.

\section{Determinación del valor de mercado de} la plantación en pie: Se determinó el valor de mercado con el cual se ajustó el valor real en pie a las condiciones objetivas de aprovechamiento y transporte de la madera de la plantación. De tal manera que la ecuación final de valor de mercado utilizada fue la siguiente:

Valor de mercado $=$ Valor real $*($ Potencial de aprovechamiento y transporte)
Los criterios que califican el potencial de aprovechamiento y transporte tienen la siguiente distribución en la ecuación global: Valor Comercial $=($ Valor Real $) *$ (Escala de operaciones*0,15 + Acceso a la plantación*0,15 + Distancia al sitio de transformación $* 0,2+$ Pedregosidad*0,05 + Densidad de siembra*0,10 + Malezas*0,05 + Topografía y pendiente*0,3).

\section{RESULTADOS Y DISCUSIÓN}

Como se observa en la tabla 2, se muestra cada uno de los criterios utilizados para el cálculo del valor de mercado de las plantaciones de teca en Córdoba, según la metodología propuesta por Murillo y Badilla (2004).

Tabla 2. Proceso de valoración de plantaciones de Tectona grandis Linn en el departamento de Córdoba, Colombia.

\begin{tabular}{|c|c|c|c|c|c|c|}
\hline \multirow{2}{*}{$\begin{array}{l}\text { Criterios de valoración } \\
\text { de las plantaciones }\end{array}$} & \multicolumn{3}{|c|}{ Canalete } & \multicolumn{2}{|c|}{ San Antero } & \multirow[t]{2}{*}{ Promedio } \\
\hline & Guazimal & Soplavientos & El Cerro & Payares & El Limón & \\
\hline Edad de la plantación (años) & 8,7 & 6,7 & 9,0 & 9,8 & 8,0 & 8,4 \\
\hline \multicolumn{7}{|l|}{ IMA $\left(\mathrm{m}^{3} \mathrm{ha}^{-1} \mathrm{año}^{-1}\right)$ del volumen trozas $1+2$} \\
\hline (2,5 $\mathrm{m}$ de largo). & $5,40 \pm 0,34$ & $2,20 \pm 0,18$ & $4,45 \pm 0,29$ & $4,11 \pm 0,26$ & $5,40 \pm 0,35$ & $4,31 \pm 0,28$ \\
\hline Población de árboles por hectárea $(\mathrm{N})$ & 669 & 885 & 1,179 & 1,019 & 1,156 & 982 \\
\hline Diámetro(cm) & 17,2 & 13,0 & 16,7 & 16,8 & 17,0 & 16,1 \\
\hline Altura (m) & 21,8 & 15,3 & 21,6 & 21,5 & 21,4 & 20,4 \\
\hline Área basal $\left(\mathrm{cm}^{2}\right)$ & 20,6 & 16,9 & 20,1 & 19,0 & 21,0 & 19,5 \\
\hline Índice de sitio & 21,0 & 17,9 & 20,3 & 19,0 & 21,6 & 20,0 \\
\hline Volumen comercial $\left(\mathrm{m}^{3} \mathrm{ha}^{-1}\right)$ & 51,7 & 12,6 & 55,7 & 36,5 & 61,1 & 43,5 \\
\hline IMA del volumen comercial (m³/ha/año) & 5,9 & 1,9 & 6,2 & 3,7 & 7,6 & 5,1 \\
\hline Valor real en pie (USD ha-1) & 12.669 & 3.097 & 13.633 & 8.945 & 14.961 & 10.661 \\
\hline \multicolumn{7}{|l|}{ Ajuste del valor real en pie por la edad del } \\
\hline árbol (USD ha-1) & 10.135 & 2.478 & 10.907 & 7.156 & 11.969 & 8.529 \\
\hline \multicolumn{7}{|l|}{ Ajuste del valor real en pie por la calidad de } \\
\hline la troza $($ USD ha-1) & 8.640 & 2.176 & 8.514 & 5.671 & 9.278 & 6.856 \\
\hline \multicolumn{7}{|l|}{ Ajuste del valor real en pie por clase } \\
\hline diamétrica de las trozas (USD ha-1) & 6.195 & 1.417 & 5.733 & 3.838 & 5.795 & 4.596 \\
\hline \multicolumn{7}{|l|}{ Valor real en pie ajustado por el potencial de } \\
\hline aprovechamiento y transporte (USD ha $\left.{ }^{-1}\right)$ & 5.482 & 989 & 4.644 & 3.108 & 4.867 & 3.818 \\
\hline
\end{tabular}


Volumen comercial en $\mathbf{m}^{3} \mathbf{h a}^{-1}$ : $\mathrm{Al}$ calcular el volumen comercial por hectárea de cada plantación se observa que no se mantiene una relación proporcional con la edad. Es así como la plantación El Limón de San Antero, presenta el mayor volumen comercial con 61,1 $\mathrm{m}^{3} \mathrm{ha}^{-1}$, a pesar de no ser la de mayor edad, debido en gran medida a que esta plantación al igual que todas las del municipio de San Antero no se les había practicado su primer raleo al momento en que fue tomada la información. Por tanto, hay un mayor número de árboles por hectárea (1.156) que aportan el volumen comercial con corteza. Otro factor que influye en el volumen por hectárea es la calidad del sitio. Debe también señalarse, que en esta plantación hay sectores con un espaciamiento mayor al del resto, esto hace disponer de mayor oferta de luz que se traduce en mejores condiciones para la fotosíntesis, que permite un mejor crecimiento de volumen de madera.

Al comparar el volumen comercial de las plantaciones del municipio de Canalete, se observa un comportamiento normal en la relación volumen comercial vs edad, mostrando a la plantación Guazimal con el mayor valor $\left(51,7 \mathrm{~m}^{3} \mathrm{ha}^{-1}\right)$ a pesar de que presenta la población más baja producto de los dos raleos que le han realizado.

Los valores del incremento medio anual (IMA) del volumen comercial se pueden considerar como bajos para la especie (Fonseca 2004), debido a la baja productividad general de estas plantaciones, que se encuentran bajo programas de manejo pobres y utilización de semilla sin mejoramiento genético.

Valor real en pie: La plantación El Limón registró el mayor valor real en pie, tal y como corresponde, por contener el mayor volumen comercial de la zona, seguida de la plantación El Cerro. Debido a que no se les ha realizado ningún raleo. Por la edad de estas plantaciones, el valor real en pie es relativamente bajo, explicado en parte por la alta proporción de albura en la madera a edades tempranas (Fonseca 2004). Según Arruda et al. (2003), en un estudio realizado en plantaciones de teca en Mato Grosso (Brasil), afirman que a las compañías forestales les resulta difícil la comercialización de la madera del primer raleo por su proporción de albura que representa el $81,19 \%$ del volumen.

Rivero (2004), encontró que en plantaciones de teca de 8 años en Bolivia el contenido de albura de las trozas estudiadas fue del $70 \%$. Murillo et al. (2011) señalan que en una troza comercial de teca se tienen dos tipos de madera, sin duramen que se vende localmente a un precio marginal $y$, con duramen que se puede vender internacionalmente con un precio elevado según sea su clase diamétrica. Por tanto, en plantaciones jóvenes la alta proporción de albura sin duda afectará su valor real.

\footnotetext{
Ajuste del valor en pie por la edad del árbol: Con este criterio se inician los ajustes del valor real en pie de la plantación. De manera general, todas las propiedades físicomecánicas y químicas de la madera son significativamente inferiores a temprana edad, ya que los árboles están todavía formando la conocida madera juvenil (Moya et al. 2009). Las condiciones ambientales de la plantación, características físico-químicas del suelo, se ha reportado que no influyen directamente en la presencia de duramen en esta especie (Moya
} 
et al. 2010). El criterio más visible es la alta presencia de albura en plantaciones jóvenes, que limita dramáticamente el potencial de venta de la madera de teca, tal y como se discutió anteriormente. Debido a que todas estas plantaciones son menores a los 10 años de edad, se les penaliza el valor real de la madera con una disminución de un $20 \%$ del valor real en pie.

\section{Ajuste del valor en pie por la calidad de las} trozas comerciales: Las piezas rectas, libres de nudos, poca conicidad, tienen sin duda un mayor porcentaje de transformación que las piezas con defectos. La plantación El Limón en San Antero, presentó la mayor disminución en su valor real debido a la baja calidad de sus trozas y árboles $(22,48 \%)$, donde solamente se registró un 3,6\% de árboles de calidad 1, la plantación de menor disminución en su valor real en pie resultó ser Soplavientos en Canalete, $(12,19 \%)$ debido a que el $77,4 \%$ de sus árboles se registraron en la calidad 1 y 2 . Al comparar las dos localidades, las plantaciones de San Antero registraron una mayor penalización por baja calidad que las plantaciones del municipio de Canalete. Si además se tiene en cuenta que las plantaciones de San Antero no han tenido una poda adecuada puede esperarse entonces que estas trozas sean de menor calidad en aserrío tal como lo afirma Epuyao (2003).

\section{Ajuste del valor en pie por la clase diamétrica}

de la troza: Como se mencionó anteriormente en la metodología, tendrá mayor valor económico una plantación con pocos árboles pero de diámetros mayores, que una plantación con muchos árboles y diámetros menores, aunque ambas registren el mismo volumen por hectárea. Esta situación se hace evidente al comparar la disminución en valor comercial de las plantaciones sin raleo, Soplavientos, El Cerro, Payares y El Limón, cuya disminución es mayor del $30 \%$ de su valor comercial original. De estas plantaciones, El Limón fue la que registró la mayor penalización $(37,54 \%)$, a pesar de mostrar el mayor volumen por hectárea. Una vez realizada la penalización por clase diamétrica, la plantación Guazimal supera a El Limón en valor, debido a que su volumen en pie es aportado por árboles con diámetros mayores que las demás plantaciones, a pesar de que mantiene la menor población de árboles por hectárea, producto de los raleos a que ha sido sometida. Estas misma razón explica que aunque Guazimal tenga menor edad que Payares la diferencia en el valor económico sea del 38\% a favor de Guazimal. Es claro entonces que la mayor disponibilidad de luz, que aumenta con la disminución de árboles por hectárea, es un factor determinante en el diámetro de las trozas. Lo que coincide con los resultados encontrados por Muñoz, et al. (2005) en Eucalyptus nitens en donde el crecimiento en diámetro fue mayor en los árboles con espaciamiento más amplio.

\section{Del valor real en pie al valor de mercado con base en la penalización por el potencial de aprovechamiento y transporte: Como último} paso en la penalización del valor en pie de la plantación, se recurre al conjunto de criterios que evalúan el potencial de aprovechamiento y transporte de la madera, con lo que finalmente se obtiene el valor de mercado. Las plantaciones de Canalete se ubican en los dos extremos en cuanto a la disminución en su valor de mercado. La plantación Soplavientos presentó una disminución en su valor de mercado del 30,2\%, mientras que 
Guazimal tuvo una disminución del 11,51\%.

Esta disminución de tanta magnitud obedece, en gran medida, a que en Soplavientos existen pendientes en el terreno que superan los $30^{\circ}$ lo que repercute directamente y negativamente en el potencial de aprovechamiento y provoca una disminución en su valor de mercado. Esta situación implica un incremento en los costos de extracción de la madera. Otros factores que incidieron en la disminución del valor de mercado de Soplavientos, aunque en menor proporción, fue el tener una área menor a 20 ha y que se requerirá la apertura de más de $1 \mathrm{~km}$ de caminos para permitir el acceso de camiones hasta el punto donde se localiza la plantacióndentro de la propiedad.

La mayor disminución del valor real en pie la obtuvo la plantación el Limón $(67,5 \%)$ a pesar de registrar el mayor volumen real de madera en pie. Esto se explica por la presencia de una alta cantidad de árboles de baja calidad (calidades 3 y 4) y que al momento del aprovechamiento de la madera generan altos desperdicios. Otro posible factor que incidió en la disminución del valor de esta plantación $(37,54 \%)$, fue el bajo diámetro promedio de sus árboles (16,38 $\mathrm{cm})$, originado por la falta de raleos oportunos. La plantación de menor disminución en su valor de mercado fue Guazimal en Canalete, con un valor de mercado final reducido en un $56,7 \%$. Proporción que supera la reportada por Murillo et al. (2004) en una plantación de pilón (Hieronyma alchorneoides) en Costa Rica, donde se registró una disminución del $40 \%$ de su valor real en pie con relación al de mercado en pie.

\section{CONCLUSIONES}

El valor real en pie de las plantaciones de teca disminuyó, por efecto de los criterios de valoración en un $64,7 \%$.

El criterio que generó mayor disminución, fue el ajuste por clase diamétrica $(33,14 \%)$ y el de menor disminución correspondió al ajuste por calidad de trozas $(18,42 \%)$.

La metodología utilizada, desarrollada en plantaciones de Costa Rica, es de fácil adaptación y uso para las condiciones de las plantaciones del departamento de Córdoba, Colombia.

\section{REFERENCIAS}

Arechederra, S. 2010. Método de Valuación de Inmuebles Históricos. Universidad Juárez Autónoma de Tabasco, México, p217.

\section{Arruda, A., Da Silvall, M., Coutoll. L. y Dias, M.} 2003. Análiseeconômica de umplantio de teca submetido a desbastes. R. Árvore, Viçosa-MG 27(4):487-494.

Barnett, J. y Jeronimidis G. (eds), 2003. Wood quality and its biological basis.Blackwell Publishing and CRC Press, p226.

Berlanga, J., Valdés, R.y Aguirre, V. 2004. Avalúo del rancho ganadero experimental Las Norias, con aplicación de los enfoques de comparación de ventas, costos e ingresos. Revista Agraria - Nueva Epoca 52(1):35-48 .

Caballer, V. 1999. "Valoración de árboles". Ed. Mundi Prensa. Madrid, p58. 
CONFEMADERAS (Confederación Española de Empresas de la Madera). 2011. Mercado de la madera en el mundo. Disponibleen la web: http://www.jovenesconmadera.com/ jovenes-con-madera/padres/el-mundoempresarial-de-la-madera/i/760/327/ mercado-de-la-madera-en-el-mundo [17 Septiembre 2011].

Corella, O. 2009. Valoración de la base forestal de las plantaciones forestales y su contribución al abastecimiento de madera en la zona del Atlántico Norte de Costa Rica. Tesis Magister Scientiaeen Manejo y Conservación de Bosques Tropicales y Biodiversidad. Escuela de Posgrado, Programa de Educación para el Desarrollo y la Conservación del Centro Agronómico Tropical de Investigación y Enseñanza (CATIE), Turrialba, Costa Rica, p86.

Cornelius, J. \& M. Hernández. 1994. Variacióngenética en crecimiento y rectitud del fusteen Gmelina arborea en Costa Rica. Boletín Mejoramiento Genético y Semillas Forestales10:9-12.

Corrales, O. 1996. Informe del estado de la industriaforestal en la zona norte de Costa Rica. Proyecto COSEFORMA. Ciudad Quesada, Alajuela, p46.

Devia, C. 2000. El desarrollo forestal como un elemento determinante para alcanzar el desarrollorural. El proyecto forestal del Valle del Río Cimitarra como estudio de caso. Seminario Internacional "La Nueva Ruralidad en América Latina", Pontificia UniversidadJaveriana. Bogotá, Colombia. Agosto de 2000.
Devia, C. 2003. Valoración de la madera en pie. Una alternativa para el manejo adecuado de los recursos forestales. Memorias del XII Congreso Forestal Mundial, FAO, Quebec, Canadá, septiembre de 2003.

Epuyao, J. 2003. Comparación del aprovechamiento y calidad de madera aserrada de Pinus radiata D. Don, entre dos tipos de trozas podadas. Tesis de Ingeniero Forestal. Universidad Austral de Chile, Valdivia, p97.

Espitia, M., O. Murillo, C. Castillo, H. Araméndiz \& N. Paternina. 2010. Ganancia genéticaesperada en la selección de acacia (Acacia mangium WILLD) en Córdoba (Colombia).Revista U.D.C.A. Actualidad \& Divulgación Científica 13:99-107.

Fonseca, W. 2004. Manual para Productores de teca (Tectona grandis L.f) en Costa Rica. Fondo Nacional de Financiamiento Forestal. San José, Costa Rica, p121.

Kumar, A., Matharoo, A. K., Singh, S. y A. N. Sing. 2006. Planting stock improvement inGmelina arborea. Indian Forester 132:691-699.

Lamprecht, H. 1990. Silvicultura nos trópicos: ecossistemasflorestais e respectivas espécies arbóreas - possibilidades e métodos de aproveitamento sustentado. Eschborn: Deustsche Gessellschaft Für Technische Zusammenarbeit (GTZ) Gmb $\mathrm{H}, \mathrm{p} 343$. 
Matricardi, A. 1989. Efeitos dos fatores do solo sobre o desenvolvimento da teca (Tectona grandis L. F.) cultivada na Grande Cáceres - Mato Grosso. 151 f. Dissertação (Mestrado em Ciências Florestais) - Escola Superior de Agricultura "Luiz de Queiroz", Piracicaba. Mesén, F. 2001. Introducción al mejoramiento genético forestal. En: Identificación, selección y manejo de fuentes semilleras. SerieTécnica /No. 32. Convenio CONIF, INSEFOR y MADR. Bogotá, p118.

Mesén, F. 2001. Introducción al mejoramiento genético forestal. En: Identificación, selección y manejo de fuentes semilleras. Serie Técnica / No. 32. Convenio CONIF,INSEFOR y MADR. Bogotá (Colombia), septiembre. ISSN 01210300, p118.

Moya, R., Berrocal, A., Serrano, J. y Tomazello, M. 2009. Variación radial de la anatomía, densidad y durabilidad de la madera de teca (Tectona grandis) procedente de dos calidades de sitio y dos regiones climáticas de Costa Rica. InvestAgrar: SistRecurFor 18(2):119-131.

Moya, R., Arce, V., González, E., Olivares C. y Rios, V. 2010. Efecto de las propiedades físicas y químicas del suelo en algunas propiedades de la madera de teca (Tectona grandis). Viçosa. Rev. Árvore34 (6):45-59.

\section{Muñoz, F., Espinosa, M., Herrera, M. y Cancino,} J. 2005. Características del crecimiento en diámetro, altura y volumen de una plantación de Eucalyptusnitens sometida a tratamientos silvícolas de poda y raleo. Bosque 26(1):93-99.

Murillo, O. 1988. Natural variation in wood specific gravityof Pinusgreggii, $P$. leiophylla and P. pringlei. Camcore. Boletín de Forestería Tropical. No. 5. School of Forest Resources.North Carolina StateUniversity. Raleigh, North Carolina, USA, p24.

Murillo, O. y Camacho P. 1997. Metodología para la evaluación de la calidad de plantaciones recién establecidas. Agronomía Costarricense 21(2):189-206.

Murillo, O. y Camacho P. 1998. Evaluación de la calidad de plantaciones forestales. Instituto Tecnológico de Costa Rica. Escuela de Ingeniería Forestal. Serie de Apoyo Académico No. 27. Cartago, Costa Rica, p56.

Murillo, O. 1999. Evaluación de la calidad en plantaciones forestales. Tatascán (Honduras) 11(1):22-36.

Murillo, O. y Badilla, Y. 2004. Evaluación de la calidad y estimación del valor en pie de la plantación forestal. Escuela de Ingeniería Forestal, ITCR. Cartago, Costa Rica, p50.

Murillo, O., Meza, A. y Cabrera, J.M. 2004. Estimación del valor real y del valor de mercado en pie de la plantación forestal. Agronomía Costarricense 28(1):47-55.

Murillo, O., Badilla, Y. y Rojas, F. 2011. Calidad de las plantaciones de teca en Costa Rica. 
En: Conferencia Forestal Internacional: Bosques plantados de teca. Teaknet. 31 octubre al 3 de noviembre, 2011. San José, Costa Rica, p22-37.

Oliveira, R. 2003. Sistema para cálculo de balanço nutricional e recomendação de calagem e adubação de povoamentos de teca - Nutriteca. 93f. Dissertação (Mestradoem Solos e Nutrição de Plantas) - Universidade Federal de Viçosa, Viçosa.

Ordóñez, J. 2012. El futuro del CIF en Colombia.http://www.revista-mm.com/ ediciones/rev76/forestal_cif.pdf Octubre 2012].

Rincón, M. 2009. El sector forestal en Córdoba: Cadena productiva forestal madera $y$ muebles departamento de Córdoba. Informe Cadena Forestal de Córdoba (Colombia), Febrero de 2009 (Centro de Investigaciones Turipaná - Corpoica), p37.

Rivero, J. 2004. Propiedades Físico-Mecánicas de Gmelina arbórea Roxb. y Tectona grandis Linn. http://www.monografias. com/trabajos16/gmelina-arborea/gmelinaarborea.shtml [17 Septiembre 2011].
Rojas, F.; Arias, D. 2004. Manual para productoresde Melina (Gmelina arborea) en Costa Rica. Cartago (Costa Rica), p86.

Rondon, N., Macedo, R. y Filho, T. 1998. Formação de povoamentos florestais com Tectona grandis L.f. (Teca). Boletim Técnico - Série Extensão 7(33):1-29.

Silva, A., Cruz, E. y Medina, P. 2010. Valoración de inmuebles compaginando teoría y práctica. Scientia et Technica Año XVI, No 45, Universidad Tecnológica de Pereira, p27.

Vallejos, J., Y. Badilla, F. Picado \& O. Murillo. 2010. Metodología para la selección eincorporación de árboles plus en programasde mejoramiento genético forestal. Agronomía Costarricense 33:105-119.

Zobel B., Van Buijtenen. 1989. Wood variation. Itscauses and control. Springer Series in Wood Science. Springer. Alemania, p363. 